\title{
Design of experiment-driven stability-indicating RP-HPLC method for the determination of tofacitinib in nanoparticles and skin matrix
}

\author{
Srividya Gorantla", Ranendra N. Saha ${ }^{1,2}$ and Gautam Singhvi ${ }^{1 *}$ (D)
}

\begin{abstract}
Background: Tofacitinib_-an oral JAK inhibitor — has been recently approved by US FDA to treat moderate to severe RA. The delivery of tofacitinib to specific inflammation site at joint via topical route using nanoformulations helps in managing the potential adverse effects. The objective is to develop and validate a simple, specific, and sensitive stability-indicating HPLC method for quantification of tofacitinib in topical nanoformulations and different matrices (adhesive tape, and skin layers, i.e., stratum corneum, viable epidermis, and dermis). The major objective was to avoid use of instruments like LC-MS/MS and to ensure a widespread application of the method.
\end{abstract}

Result: $\mathrm{A} 3^{2}$ factorial 'design of experiments' was applied to optimize process variables, to understand the effect of variables on peak properties. The calibration curve showed regression coefficient $\left(R^{2}\right) 0.9999$ and linearity in the concentration range of 50 to $15,000 \mathrm{ng} / \mathrm{mL}$, which is suitable for the analysis of conventional dosage forms and nanoformulations. Method validation was performed as per ICH guideline Q2 (R1). The accuracy by recovery studies ranged between 98.09 and $100.82 \%$. The \% relative standard deviations in intraday and interday precisions were in the range of $1.16-1.72$ and $1.22-1.80 \%$, respectively. Forced degradation studies indicated the specificity of method and showed stability-indicating potential for tofacitinib peak.

Conclusion: The validated method provides a quantification method of tofacitinib in the presence of formulation excipients, dissolution media, and skin tissues in detail. In addition, the method was successfully utilized for determination of various dermatokinetics profile of tofacitinib.

Keywords: Dermatokinetic study, Factorial design, HPLC, Nanoparticles, Tofacitinib

\section{Background}

Rheumatoid arthritis (RA) is a chronic, systemic inflammatory-mediated and pathologically complex autoimmune disease that can lead to permanent joint damage and deformity [1]. The United States Food and Drug Administration (USFDA) approved tofacitinib, the first

\footnotetext{
*Correspondence: gautam.singhvi@pilani.bits-pilani.ac.in

${ }^{1}$ Industrial Research Laboratory, Department of Pharmacy, Birla Institute of Technology and Science (BITS), Pilani, Pilani Campus, Pilani, Rajasthan 333031, India

Full list of author information is available at the end of the article
}

Janus kinase (JAK) inhibitor, in November 2012 for the treatment of moderate to severe RA. Tofacitinib is a selective small-molecule inhibitor of JAK3 and JAK1, and tyrosine kinase (TYK2) to a lesser extent [2]. Importantly, tofacitinib can also modulate T-cell activation, synovitis, and structural joint damage [3]. Tofacitinib is taken twice a day orally in doses of $5 \mathrm{mg}$ [4]. Oral administration of tofacitinib in high doses (5 $\mathrm{mg}$ and $10 \mathrm{mg}$ per day) suppresses the immune system and can make the subject susceptible to infections. Thus, there is a need to explore other routes of administration for effective delivery of tofacitinib. Recently, topical application of $2 \%$ tofacitinib 
ointment has shown good therapeutic activity for treating psoriasis condition in clinical trials [5]. The topical administration of tofacitinib can overcome the limitations of oral therapy, such as pre-systemic metabolism, gastrointestinal problems, dose escalation, and nontissue distribution and systemic side effects (decrease in neutrophils count). Moreover, compared to the oral route, the topical and transdermal routes require a low dose of tofacitinib, attenuating systemic side effects.

In the case of topical and transdermal delivery, the outermost layer on the skin (stratum corneum) acts as a barrier [6]. The stratum corneum consists of corneocytes in the intercellular matrix form a barrier for the permeation of drugs through the skin. Therefore, lipid-based nanocarriers, viz. solid lipid nanoparticles (SLNs), have been designed to enhance the permeation of drug through the skin [7]. Further, the formulated lipid nanocarriers need to be evaluated for entrapment efficiency, drug release, and permeation through skin layers. Most importantly, to evaluate the topical drug delivery, there is a need to assess the permeation of tofacitinib through different layers of the skin (dermatokinetics). Thus, to understand the dermatokinetics, it is essential to develop a method for determination of tofacitinib in the skin tissues.

A thorough literature survey revealed that there are few tandem mass spectrometry (LC-MS/MS) [8-14], and matrix-assisted laser desorption ionization mass spectrometry imaging (MALDI-MS) [15] methods are available for the estimation of tofacitinib alone and in combination with other analytes. These mass spectrometry methods are sensitive, but the routine estimation of in vitro release samples, and stability samples would be difficult and expensive when the number of samples is more. Additionally, these methods involved very complicated procedures and required additional sample treatment.

The reported HPLC methods for tofacitinib determination showed lower limit of quantification in microgram level only and not fully validated as per ICH guidelines with respect to stability studies and robustness $[8,16-$ 23]. The reported spectrometric methods are economi$\mathrm{cal}$ and adequate for the estimation of tofacitinib in pure form and in its dosage forms [24, 25]. However, these spectrometric methods were not explored for nanogram sensitivity, purity of target peak, and stability. Recently, a fully automated in situ ultraviolet fiber optic system with 10-mm-arch probes for the estimation of TF has been reported [26]. However, this is quite expensive, require accurate installation, a trained operator, and it might be difficult to design acceptable measurement systems for routine analysis. Further, the above reported methods were not investigated and validated in the presence of skin tissue or for complex nanoformulations.
Therefore, in the current work, we developed and validated a rapid, accurate, and precise stability-indicating reverse-phase HPLC method for the estimation of tofacitinib up to 50 nanogram levels in nanocarrier formulation and skin tissues. Further, the design of experiment (DoE) was applied to study the factor interaction and their impact on chromatographic properties [27]. The developed method is suitable for industry and academia for quantification of tofacitinib in nanoformulations, with ease and economic way. It is employed for evaluation of entrapment efficiency, stability, and in vitro release profile of tofacitinib-loaded SLNs. The $\%$ recovery of tofacitinib in the skin matrix was calculated, and the method was successfully applied for studying the dermatokinetics of tofacitinib on topical application.

\section{Methods}

\section{Solvents and chemicals}

Methanol and acetonitrile (HPLC grade) were acquired from Merck Limited, Mumbai (India). Ammonium acetate, potassium phosphate monobasic, orthophosphoric acid, and tetra-hydrofuran were acquired from Merck. Milli-Q water was attained in-house from a Milli-Q water purification system, Millipore (USA).

\section{Instrumentation and chromatographic conditions}

The chromatographic experiments were conducted on the HPLC system (Shimadzu, Kyoto, Japan) comprised of model LC-10AT, a binary pump, SIL-HTA autosampler (Shimadzu, Kyoto, Japan), column oven (CTO10AS) compartment, and SPD-M20A photodiode array (PDA) detector. Chromatographic separation was carried out at $30 \pm 0.5{ }^{\circ} \mathrm{C}$ using LiChrospher ${ }^{\mathrm{R}} 100 \mathrm{RP}-18$ analytical column $\left(\operatorname{Hibar}^{\mathrm{R}} 250-4.6 ; 5 \mu \mathrm{m} ; \mathrm{Merck}^{\mathrm{R}}\right)$. In the first instance, preliminary trials were conducted to acquire knowledge about the method performance and identification of various critical, independent parameters, and its effect on dependent variables. Systematic method development strategies were applied to identify the independent parameters with less number of trials. Primarily, acetonitrile and methanol were tried with various \% ratios of ammonium acetate and phosphate

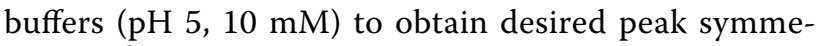
try. A $3^{2}$ full factorial experiment design was applied for the optimization of the mobile phase composition. The detection was carried out with a PDA detector with $30 \mu \mathrm{L}$ injection volume at wavelength $285 \mathrm{~nm}$. The data acquisition and HPLC system were controlled by the LC solution software version 1.24 SP1. 


\section{Methods}

Preparation of stock, calibration, and quality control standards

A stock solution of tofacitinib $(1 \mathrm{mg} / \mathrm{mL})$ was prepared by dissolving an accurately weighed amount of tofacitinib in methanol. The working standard solution $(100 \mu \mathrm{g} / \mathrm{mL})$ and calibration standards 50-15,000 ng/ $\mathrm{mL}$ were prepared through serial dilution with methanol. The quality control (QC) standards were prepared from the standard stock at three concentration levels: low QC (250 ng/mL), medium QC (8000 ng/mL), high QC $(12,000 \mathrm{ng} / \mathrm{mL})$, and the lower limit of quantification (LLOQ) $(50 \mathrm{ng} / \mathrm{mL})$.

\section{DoE methodology and optimization of analytical method}

Using the above-executed trials, the organic phase and
Linearity, limit of detection and quantification limit

Linearity was determined with a concentration range between 50 and $15,000 \mathrm{ng} / \mathrm{mL}$ with six calibration standards. The obtained data were fitted into linear regression analysis, and the calibration curve was plotted by the analyte peak area on the $x$-axis against the concentration of analyte on the $y$-axis. The detection limit (or) limit of detection (LOD) and quantification limit (or) limit of quantification (LOQ) were decided based on the signal-to-noise $(S / N)$ ratio. Initially, in the system suitability test, the signal-to-noise ratio was obtained based on the detector response. The preferred $S / N$ ratios were 3:1 and 10:1 for LOD and LOQ, respectively. LOD and LOQ were calculated based on the below mentioned formula [29]. From these, LLOQ has been determined and considered as the lowest standard of the calibration curve.

$$
\text { LOD and LOQ }=\frac{\text { Concentration of standard }}{\frac{S}{N} \text { value of standard }} * \text { Desired } \frac{S}{N} \text { value }
$$

$\mathrm{pH}$ of phosphate buffer demonstrated a high impact on retention time and tailing factor. Therefore, to investigate the effect of organic phase composition and phosphate buffer $\mathrm{pH}$ on drug retention time and tailing factor, DoE methodology was applied [28]. A $3^{2}$ factorial design consisting of 2 factors at 3 levels was considered for an experimental plan with Design-Expert 8.0 Stat-Ease Inc. Minneapolis, USA. The two independent variables \% organic phase $\left(X_{1}\right)$, and $\mathrm{pH}$ of phosphate buffer $\left(X_{2}\right)$ with 3 levels $(-1$ (3.5), $0(4.5),+1$ (5.5)) were confiscated as the actual value. The retention time and tailing factor were considered as dependent variables as responses $Y_{1}$ and $Y_{2}$, respectively.

\section{Validation of the developed method}

The validation of the analytical method was performed for system suitability, linearity, range, detection limit, quantification limit, specificity, accuracy, precision, carryover effect, and robustness according to the $\mathrm{ICH}$ Q2 (R1) guideline (2005).

\section{System suitability}

System suitability test was preferred for chromatographic methods to ensure that the system is efficient to give reproducible results. The performance of the system was evaluated by injecting six replicates of $10 \mu \mathrm{g} /$ $\mathrm{mL}$ concentration with optimized chromatographic conditions.

\section{Accuracy and precision}

The measurement of the observed value's proximity to a given value is known as accuracy. Precision, on the other hand, relates to the closeness of measurement values to one another. The accuracy and precision of the quality control samples LQC, MQC, and HQC, as well as LLOQ, were determined in six replicates on intra- and interdays. Accuracy was denoted as \% bias and the precision as \% relative standard deviation (RSD). The acceptance requirement for precision and accuracy of quality control samples according to regulatory criteria was $\leq \pm 2 \%$ and $\leq \pm 10 \%$ RSD, respectively [30].

\section{Carryover effect}

The carryover was assessed by analyzing successive samples $(10 \mu \mathrm{g} / \mathrm{mL}, 12 \mu \mathrm{g} / \mathrm{mL}$, and $15 \mu \mathrm{g} / \mathrm{mL})$ of the linearity curve followed by a blank. Carryover acceptance criteria should not be higher than $20 \%$ of LLOQ.

\section{Robustness}

Robustness can be defined as the reproducibility potential of the developed method in the same laboratory conditions with slight modification in chromatographic conditions and different HPLC systems with specified conditions. Initially, the robustness of the developed analytical method was performed by changing the column oven temperature, to $\pm 5{ }^{\circ} \mathrm{C}$, and mobile phase $\mathrm{pH}$, to \pm 0.5 . Further, the optimized method was tested on another laboratory condition with the Shimadzu system (model number: LC 2010CHT). 


\section{Specificity}

The specificity of the developed analytical method was studied in the presence of formulation excipients (lipids). A known concentration of tofacitinib within the linearity range was spiked into the lipids and analyzed using the developed HPLC method. The interference of lipids with the retention time of analyte and peak purity was observed.

\section{Stability-indicating property of the developed method}

The stability-indicating property of the developed analytical method was studied by exposing the tofacitinib solution to stress conditions as per ICH Q1A (R2) guidelines. The stress studies of tofacitinib solution were conducted under acidic hydrolysis, base hydrolysis, oxidation, and thermolytic conditions [31]. The acid hydrolysis and base hydrolysis were carried out by preparing the tofacitinib solution $(200 \mu \mathrm{g} / \mathrm{mL})$ using $0.5 \mathrm{M}$ Hydrochloric acid and $0.5 \mathrm{M}$ sodium hydroxide and kept for reaction on a water bath at $60{ }^{\circ} \mathrm{C}$ for $6 \mathrm{~h}$. Similarly, for oxidative degradation, tofacitinib solution was prepared using $2 \%$ hydrogen peroxide and heated at $60{ }^{\circ} \mathrm{C}$ under reflux condition for $3 \mathrm{~h}$. For thermal degradation, the tofacitinib solution was heated at $80^{\circ} \mathrm{C}$ under reflux condition for $6 \mathrm{~h}$. After subjecting tofacitinib solution $(200 \mu \mathrm{g} / \mathrm{mL})$ to the abovesaid stress conditions, a concentration of $10 \mu \mathrm{g} / \mathrm{mL}$ was prepared using methanol. The samples of acid and base were neutralized before dilution with methanol to protect the column. All the samples were filtered through a $0.2-\mu \mathrm{m}$ filter before injecting it into HPLC analysis [32, 33]. The chromatogram of different stress conditions was recorded and compared with the normal condition. The retention time of different degradant peaks and drug peaks was identified and calculated the $\%$ degradation.

\section{Applicability of the developed method for skin studies of tofacitinib and dermatokinetic assessment}

The validated method for quantification of tofacitinib in the presence of skin tissue matrix was used to evaluate tofacitinib penetration through skin. Initially, skin tissue was homogenized using an Ultra-Turrax type homogenizer. Furthermore, the homogenized tissue was spiked with a predetermined concentration of tofacitinib solution $(500 \mu \mathrm{g} / \mathrm{mL})$ and centrifuged.. The supernatant $(1 \mathrm{~mL})$ was collected, and from this a concentration of $10 \mu \mathrm{g} / \mathrm{mL}$ was prepared. The samples were filtered through the $0.22-\mu$ membrane filter before analysis and observed for peak specificity, and percent recovery was calculated [34]. Inhouse tofacitinib cream was prepared using stearic acid as base $(0.5 \mathrm{mg} / \mathrm{g})$ and applied topically $(350 \mathrm{mg})$. After topical application, skin samples were collected for each time point at 2, 4, 6, 8, 12, $24 \mathrm{~h}$. The collected skin samples were washed with phosphate buffer and gently wiped with the cotton. Tape stripping analysis was performed to separate the epidermis and dermis layers. The collected tapes and skin were soaked for $6 \mathrm{~h}$ in methanol. After $6 \mathrm{~h}$, the samples were filtered through $0.25-\mu$ filter and then analyzed at $-20{ }^{\circ} \mathrm{C}$ until analyzed as per validated method. Tofacitinib concentration in epidermis and dermis-time profiles were analyzed by non-compartmental model approach to determine $t_{1 / 2}$, half-life; $\mathrm{C}_{0}$, tofacitinib concentration in epidermis and dermis at $t=0 ; \mathrm{AUC}_{0-t}$, area under the curve from zero to the last measurable point; $\mathrm{AUC}_{0-\infty}$, area under curve from time 0 extrapolated to infinity.

\section{Applicability in characterization of nanoformulation}

The validated analytical method was solicited for its use in the characterization of SLNs. The tofacitinib-loaded SLNs were prepared by the hot emulsification technique $[35,36]$. The SLNs were prepared using Precirol as solid lipid and Poloxamer 407 as a surfactant as per the reported method. The prepared formulation was analyzed for its entrapment efficiency, in vitro drug release ( $\mathrm{pH} 7.4)$, and stability studies.

\section{Entrapment efficiency}

The entrapment efficiency of prepared SLNs formulation was estimated by indirect method. In brief, the formulation was subjected to ultracentrifugation using Remi cooling centrifuge (Mumbai, India). The clear supernatant was diluted with the methanol and analyzed by the validated method.

\section{\% Entrapment Efficiency \\ $=1-\frac{\text { Free tofacitinib in nanocarrier dispersion }}{\text { Total tofacitinib in nanocarrier dispersion }} * 100$}

\section{In vitro drug release studies}

The in vitro release of tofacitinib from SLNs dispersion was estimated using the dialysis bag technique. The study was performed using $\mathrm{pH} 7.4$ phosphate buffer with $0.15 \%$ sodium lauryl sulfate. A known amount of formulation was transferred into the dialysis bag and maintained at $32 \pm 0.5^{\circ} \mathrm{C}$. The samples were collected at regular intervals $1,2,4,6 \mathrm{~h}$ and replaced with the fresh buffer to maintain sink condition. The tofacitinib concentration was assessed using an approved method after the samples were filtered through a $0.22-\mathrm{m}$ filter.

\section{Stability studies}

Pharmaceutical formulation development involves stability at storage conditions to maintain drug activity. Thus, short-term stability of prepared SLNs formulation was carried out at room temperature and controlled condition for 
three months. The formulation was tested for entrapment efficiency after three months of storage period using the validated analytical method.

\section{Results}

DoE methodology and optimization of analytical method

Preliminary studies were performed for the development of HPLC method. Initially, different combinations of mobile phases were explored using methanol, acetonitrile, and aqueous buffers (potassium phosphate and ammonium acetate $-10 \mathrm{Mm}$ ). Peak splitting and broad peaks were observed in the case of acetonitrile, whereas peak symmetry was found acceptable in the case of methanol. Methanol was combined with different proportions of aqueous buffers, and chromatogram properties (peak area, tailing factor, and retention time) were observed. The peak properties were analyzed, and it was observed that methanol in combination with $10 \mathrm{mM}$ phosphate buffer showed good peak properties. The above-mentioned preliminary studies were performed with the aqueous mobile phase in 50:50 v/v. The injection volumes of $20 \mu \mathrm{L}$ and $30 \mu \mathrm{L}$ were screened, and it was found that $30 \mu \mathrm{L}$ showed good peak area with reduced tailing factor. The above trials revealed that the peak properties were mostly influenced by the methanol percentage and $\mathrm{pH}$ of the aqueous phosphate buffer. Further, to optimize these parameters, full factorial designed was applied.

A total of 11 experimental runs were performed based on $3^{2}$ factorial design, and obtained results were analyzed for the retention time and tailing factor. The results of the responses are specified in Table 1 . To evaluate the relation between the dependent and independent variables, the response surface methodology plots were produced

Table 1 Suggested experiments by design of experiments and responses

\begin{tabular}{llll}
\hline $\begin{array}{l}\text { Variable 1 } \\
\text { Organic phase }\end{array}$ & $\begin{array}{l}\text { Variable } \mathbf{2} \\
\text { Buffer } \mathbf{~ H ~}\end{array}$ & $\begin{array}{l}\text { Response 1 } \\
\text { Retention time }\end{array}$ & $\begin{array}{l}\text { Response 2 } \\
\text { Tailing factor }\end{array}$ \\
$\mathbf{\%}$ & - & Min & - \\
\hline 40 & 3.5 & 7.243 & 1.681 \\
50 & 3.5 & 4.918 & 1.514 \\
60 & 3.5 & 3.667 & 1.325 \\
40 & 4.5 & 9.790 & 1.652 \\
50 & 4.5 & 5.430 & 1.473 \\
60 & 4.5 & 3.868 & 1.352 \\
40 & 5.5 & 11.168 & 1.435 \\
50 & 5.5 & 5.630 & 1.365 \\
60 & 5.5 & 3.902 & 1.334 \\
50 & 4.5 & 5.420 & 1.472 \\
50 & 4.5 & 5.430 & 1.455 \\
\hline
\end{tabular}

from Design expert software, as reported in Fig. 1. The experimental runs' responses were fitted into linear, second-order, and quadratic models. Quadratic models proved to be the best fit with $p<0.0001$. The best fit model was found to be quadratic models $(p<0.0001)$. The model summary statistics suggested the quadratic model for responses, because of the low prediction error sum of squares (PRESS) value of the quadratic model. Here, the low standard deviation, high adjusted $R^{2}$ value, specifies the good correlation of the fitted model with experimental data. The model was analyzed and validated by analysis of variance (ANOVA), and the results are shown in Table 2.

The independent variables exhibited a reasonable impact on retention time. ANOVA analysis (Table 2) showed model $F$-value of 80.51 ( $p$ value $<0.0001$ ), stipulated that the model was significant. The $3 \mathrm{D}$ plots, $2 \mathrm{D}$ contour plots, and final polynomial equation (1) for actually coded variables showed the relationship between $\%$ of the organic phase $\left(X_{1}\right)$ and $\mathrm{pH}$ of phosphate buffer $\left(X_{2}\right)$, on the retention time $\left(Y_{1}\right)$ (Fig. 1). Figure 1a, b shows that the methanol ratio in the mobile phase had shown a notable effect on retention time. The retention time was decreased with an increase in methanol percentage in mobile phase composition. In contrast, increasing the $\mathrm{pH}$ of phosphate buffer resulted in increased retention time. The combination of both the variables $X_{1}$ and $X_{2}$ showed a parabolic effect on the retention time. The obtained polynomial equation (1) has confirmed the same.

The polynomial equation (1) showed that the \% of the organic phase $\left(X_{1}\right)$ showed a negative effect while $\mathrm{pH}$ of phosphate buffer $\left(X_{2}\right)$ showed a positive effect, but the combination of both the independent variables exhibited a negative effect on retention time $\left(Y_{1}\right)$.

$$
\text { Retention time } \begin{aligned}
\left(Y_{1}\right)= & +22.67376-1.17143 X_{1} \\
& +7.65975 X_{2}-0.092250 X_{1} X_{2} \\
& +0.013072 X_{1}^{2}-0.24834 X_{2}^{2}
\end{aligned}
$$

The independent variables showed a reasonable impact on the tailing factor. ANOVA analysis showed model $F$-value of 50.03 with $p$ value 0.0003 , indicating that the model was significant (Table 2). The 3D plots and final polynomial equation (2) for actual coded variables showed the relationship between $\%$ of the organic phase $\left(X_{1}\right)$ and $\mathrm{pH}$ of phosphate buffer $\left(X_{2}\right)$, on the tailing factor $\left(Y_{2}\right)$ (Fig. 1a, b).

Figure 1c, $d$ shows that the methanol ratio in the mobile phase had shown a prominent effect on the tailing factor. The obtained polynomial equation (2) has proved the same.

The polynomial equation (2) showed that the $\%$ of the organic phase $\left(X_{1}\right)$ showed a negative effect, while the 


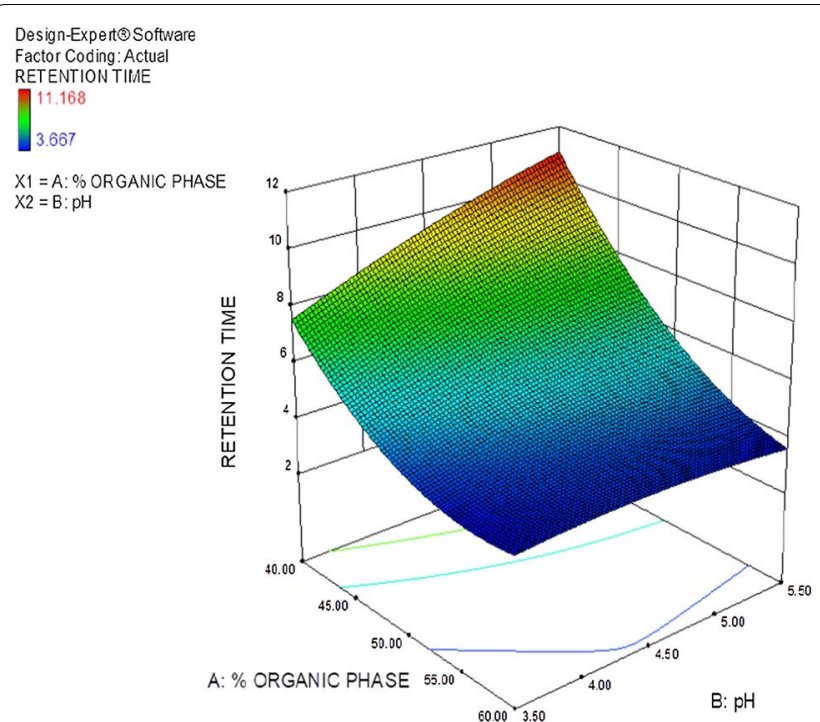

(a)

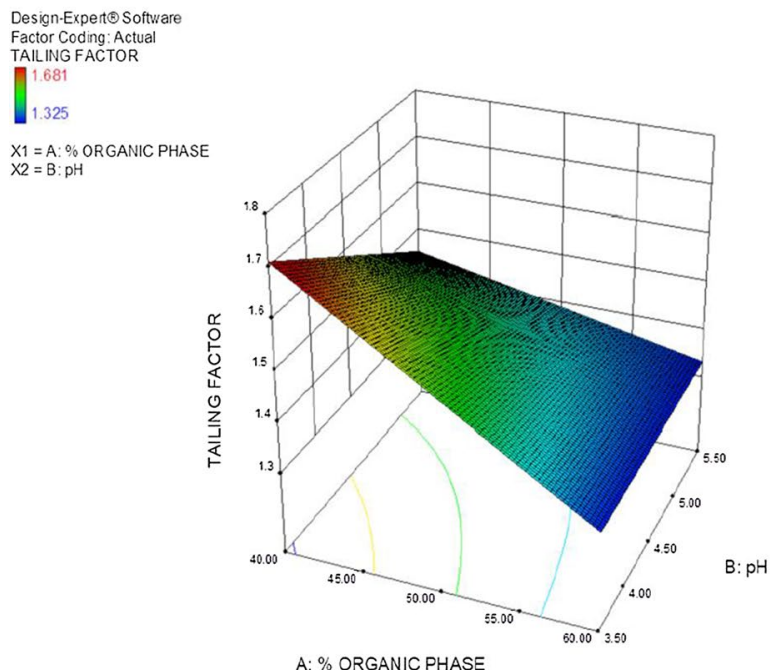

(c)
Design-Exper $\circledast$ Softwar Factor Coding: Acturar

RETENTIONT

3.11 .16

$\mathrm{X} 1=\mathrm{A}: \%$ ORGANIC PHASE $\mathrm{X} 2=\mathrm{B}: \mathrm{pH}$

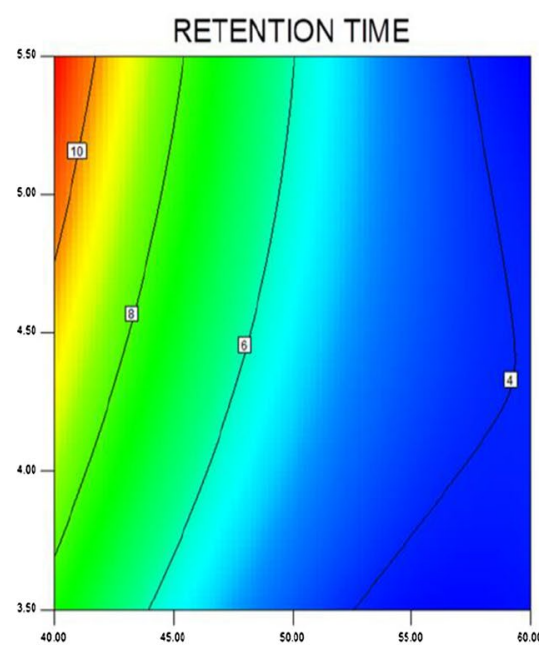
X1: A: \% ORGANIC PHASE
X2: B: $p H$

(b)
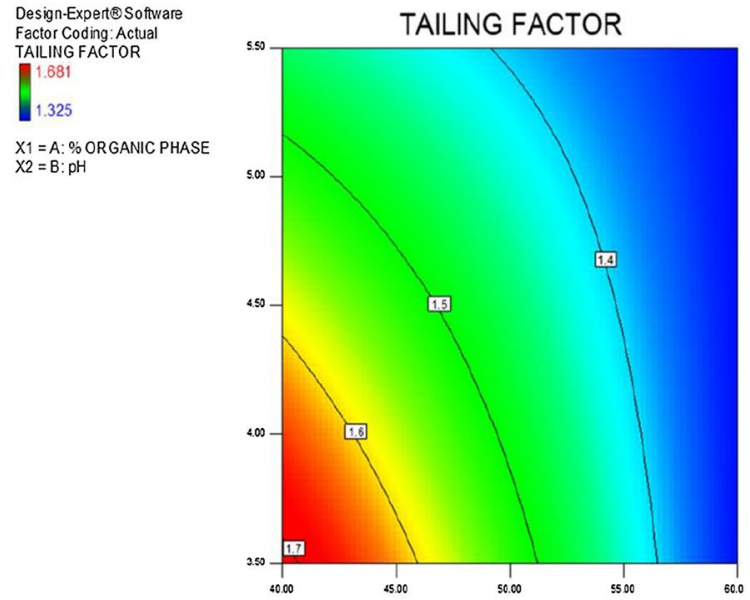

X1: A: \% ORGANIC PHASE $\mathrm{X} 2 \mathrm{~B}: \mathrm{pH}$

(d)

Fig. 1 a, b represent the 3D response curve and 2D contour plots for response 1, i.e., retention time; $\mathbf{c}$, $\mathbf{d}$ represent the 3D response curve and 2D contour plots for response 2 , i.e., tailing factor

$\mathrm{pH}$ of phosphate buffer $\left(X_{2}\right)$ exhibited a positive effect, but the combination of both the independent variables exhibited a positive effect on the tailing factor $\left(Y_{2}\right)$.
The $3^{2}$ factorial design presented 50 solutions for the optimized chromatographic conditions, but the solutions were reduced by setting the goal values. The optimized

Tailing factor $\left(Y_{2}\right)=+3.42085-0.060278 X_{1}$

$$
\begin{aligned}
& +8.65351 \mathrm{E}-003 X_{2}+6.37500 \mathrm{E}-003 X_{1} X_{2} \\
& +1.89737 \mathrm{E}-004 X_{1}^{2}-0.043526 X_{2}^{2}
\end{aligned}
$$


Table 2 ANOVA for response surface quadratic model

\begin{tabular}{lcc}
\hline Parameter & $\begin{array}{l}\text { Response 1 (Retention } \\
\text { time) }\end{array}$ & $\begin{array}{l}\text { Response } \\
\text { 2 (Tailing } \\
\text { factor) }\end{array}$ \\
\hline Std. Dev. & 0.38 & 0.024 \\
Mean & 6.04 & 1.46 \\
C.V. $\%$ & 6.31 & 1.63 \\
R-squared & 0.9877 & 0.9804 \\
Adj $R$-squared & 0.9755 & 0.9608 \\
Pred $R$-squared & 0.8712 & 0.8210 \\
Adeq precision & 26.389 & 21.688 \\
Fvalue & 80.51 & 50.03 \\
$p$ value & $<0.0001$ & 0.0003 \\
\hline
\end{tabular}

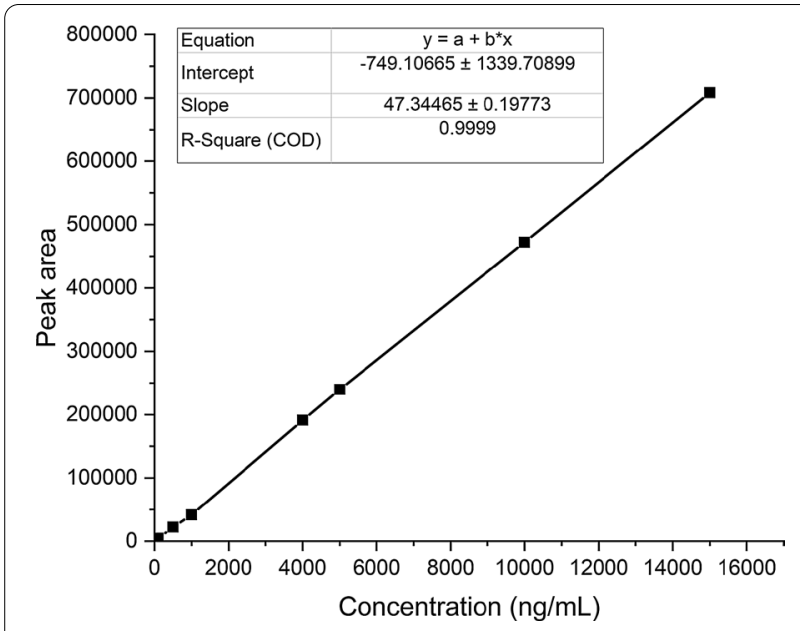

Fig. 2 Calibration curve of tofacitinib

chromatogram conditions were found to be methanol and $10 \mathrm{mM}$ phosphate buffer ( $\mathrm{pH} 3.5)$ in the ratio of $50: 50 \% \mathrm{v} / \mathrm{v}$ with a flow rate of $0.8 \mathrm{~mL} / \mathrm{min}$.

\section{Validation of the developed method System suitability}

The system suitability was estimated by six replicate injections of $10 \mu \mathrm{g} / \mathrm{mL}$ concentration of tofacitinib with the optimized chromatographic conditions. The tailing factor was found to be 0.997, which indicated the acceptability of peak properties. The \% RSD for peak area was within $\pm 2 \%$ indicated the suitability of the system. The standard calibration curve of tofacitinib was constructed. The linearity between the concentration of tofacitinib and peak area was obtained in the range of $50 \mathrm{ng} / \mathrm{mL}$ to $15 \mu \mathrm{g} / \mathrm{mL}$ with an excellent regression coefficient of 0.999 . The obtained linear regression coefficient was $y=47.34 x-749.10$. In the regression equation, ' $x$ ' is the concentration of tofacitinib and ' $y$ ' is the peak area at
$285 \mathrm{~nm}$. The calibration curve of tofacitinib is presented in Fig. 2. The LOD and LOQ of the developed analytical method were determined using the signal-to-noise ratio, and values were found to be 16.5 and $49.5 \mathrm{ng} / \mathrm{mL}$, respectively. The LLOQ was found to be $50 \mathrm{ng} / \mathrm{mL}(n=6)$. The results proved that the developed method was sensitive enough to detect and quantify tofacitinib in nanogram levels. This method would be beneficial for routine analysis of tofacitinib in nanoformulation where the entrapped drug is in low concentration.

\section{Accuracy and precision}

The accuracy and precision were estimated by standard addition method with three concentrations of LQC (250 ng/mL), MQC (8000 ng/mL), and HQC (12,000 ng/ $\mathrm{mL}$ ). The chromatograms representing LQC and HQC are presented in Fig. 3. The method demonstrated acceptable \% recovery and reproducibility with \% RSD and \% bias less than $2 \%$. Intra- and inter-day accuracy and precision data of tofacitinib are depicted in Table 3.

\section{Carryover effect}

The carryover effect was estimated by analyzing successive samples, i.e., $10 \mu \mathrm{g} / \mathrm{mL}, 12 \mu \mathrm{g} / \mathrm{mL}$, and $15 \mu \mathrm{g} / \mathrm{mL}$ concentration of tofacitinib followed by blank sample. There was no tofacitinib peak observed in the blank at the retention time of $6.1 \mathrm{~min}$. The result demonstrated that no carryover effect was observed; thus, this method could be utilized for an unremitting run for more number of samples.

The optimized chromatographic conditions were evaluated for robustness with the deliberate change in oven temperature and mobile phase $\mathrm{pH}$. The effect was found to be insignificant with these two variables on analysis of LQC, MQC, and HQC. The RSD (\%) was less than 2 and showed good reproducibility of peak properties. The obtained result implied that the developed method was stable against small variations in intrinsic parameters.

\section{Robustness}

The robustness of the analytical method was performed on another Shimadzu system (model number: LC 2010CHT) with the optimized chromatogram conditions. The results demonstrated that there was no change in peak area and tailing factor. The observed changes were insignificant; hence, this method could be easily transferable from one laboratory to another laboratory.

\section{Specificity}

The specificity study was performed with the presence of lipids used for SLNs preparation. There was no peak interference observed at the retention time of the 


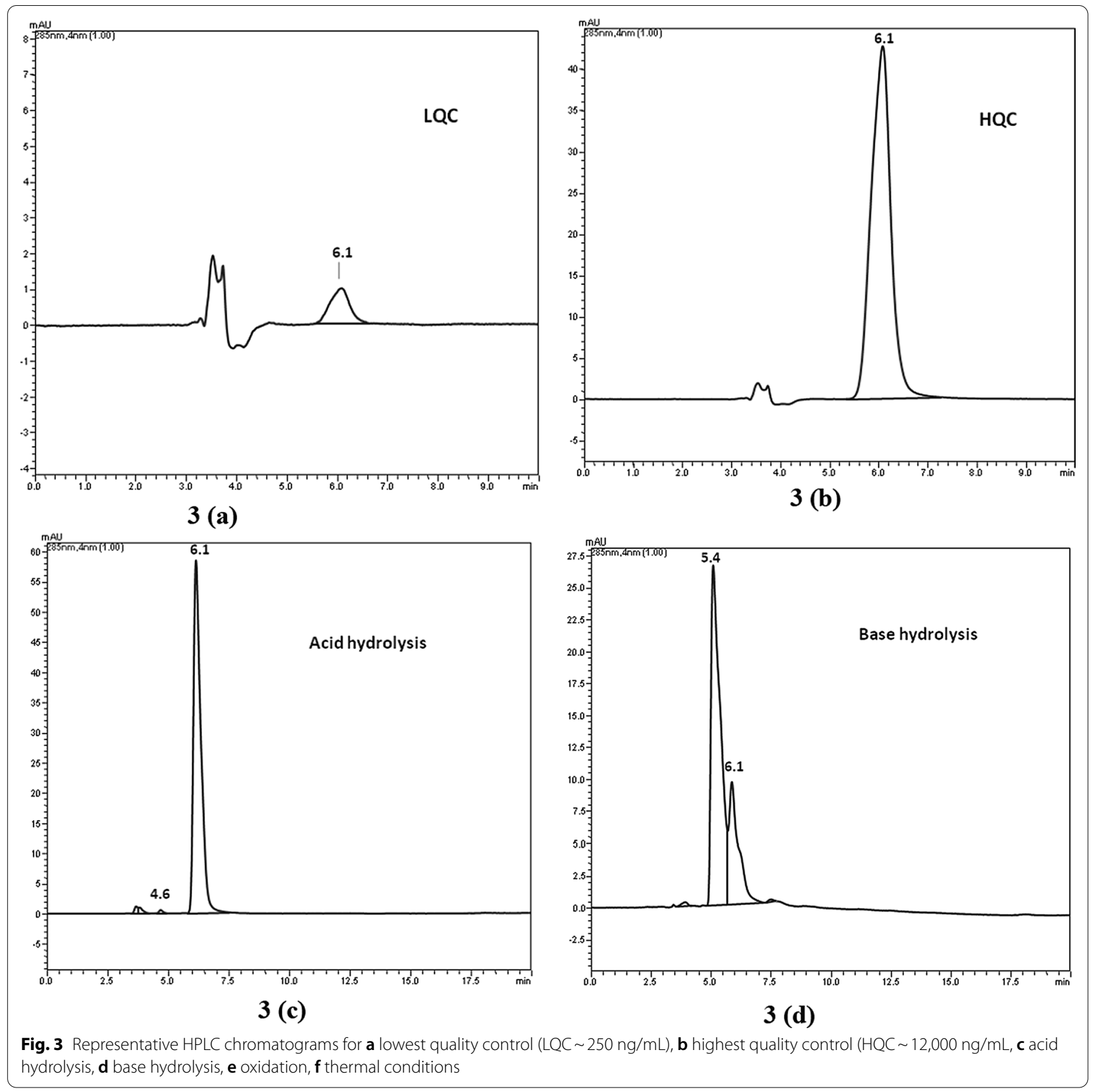

tofacitinib peak, and the purity of peak was found to be $99.99 \%$. The forced degradation studies such as acid hydrolysis, base hydrolysis, oxidation, and thermal were successfully carried out. The stability results of tofacitinib are shown in Table 4. The chromatogram of acid hydrolysis (Fig. 3c) sample of tofacitinib showed degradation peaks at 3.67, 3.8, and $4.6 \mathrm{~min}$. There was $14.78 \%$ degradation of tofacitinib observed under acidic hydrolysis. The chromatogram of base hydrolysis (Fig. 3d) sample of tofacitinib showed peak splitting, and the impurity peak was prominent and eluted at the retention time of 5.5 . This indicates that nearly $75 \%$ of tofacitinib was degraded. The observed base degradation result was confirmed with the reported study by Younis et al. They reported that above the $\mathrm{pH} \sim 9$, tofacitinib citrate showed the highest degree of degradation [37]. In contrast, under oxidative stress conditions, the chromatogram (Fig. 3e) of tofacitinib generated impurity peaks at 3.7 and $4.6 \mathrm{~min}$, and $20.25 \%$ degradation of tofacitinib was observed. The thermal degradation samples of tofacitinib did not show 


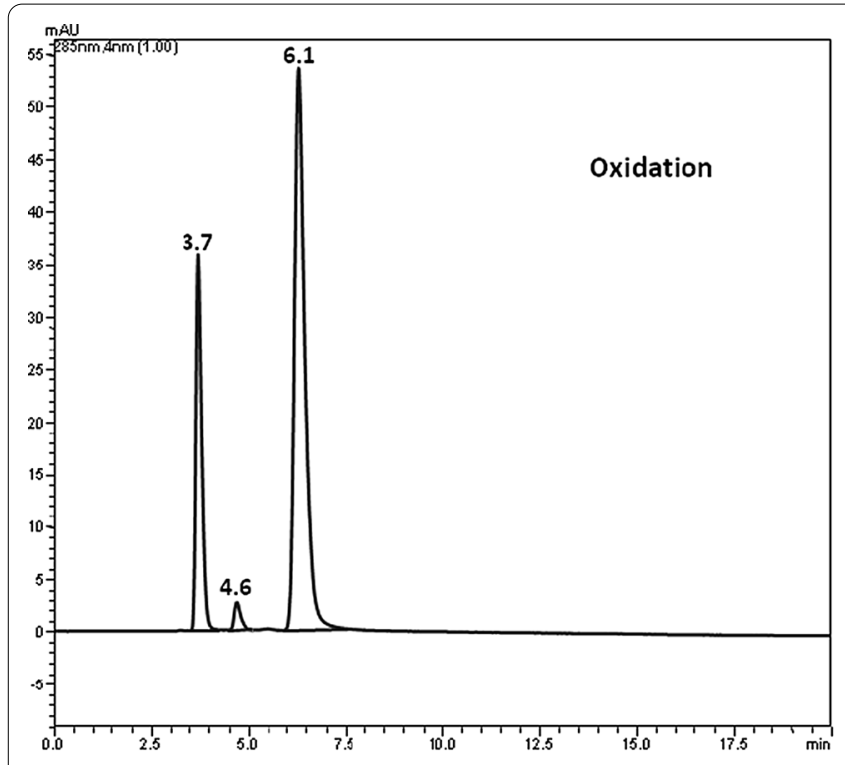

3 (e)

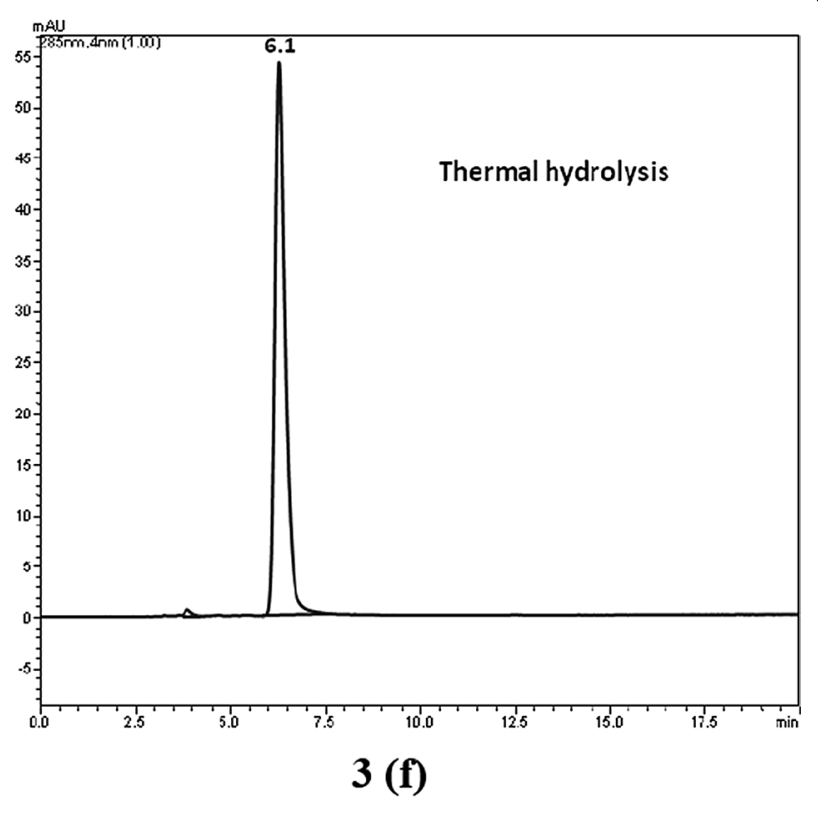

Fig. 3 continued

Table 3 Intra- and inter-day precision and accuracy results $(n=6)$

\begin{tabular}{|c|c|c|c|c|}
\hline \multirow{2}{*}{$\begin{array}{l}\text { Sample } \\
\text { QC }\end{array}$} & \multicolumn{2}{|l|}{ Intraday precision } & \multicolumn{2}{|l|}{ Accuracy } \\
\hline & $\begin{array}{l}\text { Experimental concentration }(\mu \mathrm{g} / \\
\mathrm{mL})\end{array}$ & $\%$ Recovery \pm SD & $\%$ RSD & $\%$ Bias \\
\hline LQC & $252.06 \pm 4.32$ & $100.81 \pm 1.73$ & $1.1666 \pm 0.019$ & $0.818 \pm 0.39$ \\
\hline MQC & $7847.82 \pm 192.2$ & $98.09 \pm 1.52$ & $1.7295 \pm 0.0423$ & $1.908 \pm 0.20$ \\
\hline \multirow[t]{2}{*}{$\mathrm{HQC}$} & $11,781.5 \pm 213.9$ & $98.17 \pm 1.25$ & $1.2827 \pm 0.023$ & $1.827 \pm 0.56$ \\
\hline & Interday precision & & & \\
\hline LQC & $252.06 \pm 4.32$ & $100.82 \pm 1.44$ & $1.6498 \pm 0.027$ & $0.824 \pm 0.39$ \\
\hline MQC & $7915.25 \pm 96.84$ & $98.94 \pm 1.35$ & $1.2219 \pm 0.014$ & $1.059 \pm 0.20$ \\
\hline $\mathrm{HQC}$ & $11,797.79 \pm 212.75$ & $98.31 \pm 1.43$ & $1.8017 \pm 0.032$ & $1.685 \pm 0.43$ \\
\hline
\end{tabular}

LQC: $250 \mathrm{ng} / \mathrm{mL}$; MQC: $8000 \mathrm{ng} / \mathrm{mL}$; HQC: $12,000 \mathrm{ng} / \mathrm{mL}$

Table 4 Stability results of tofacitinib in forced degradation study

\begin{tabular}{llcc}
\hline Study performed & Stress condition & \% of Tofacitinib degraded & $\begin{array}{c}\text { Degradation peaks } \\
\text { with retention time } \\
\text { (min) }\end{array}$ \\
\hline Acid hydrolysis & & & $3.67,3.8$ and 4.6 \\
Base hydrolysis & 0.5 M Hydrochloric acid & $14.78 \pm 0.04 \%$ & 5.4 \\
Oxidative & 0.5 M sodium hydroxide & $75 \pm 0.06 \%$ & 3.7 and 4.6 \\
Thermal & $2 \%$ hydrogen peroxide & $20.25 \pm 0.04 \%$ & - \\
\hline
\end{tabular}

any impurity peak (Fig. 3f). The sample showed $1.5 \%$ degradation of tofacitinib.

The results confirmed that the developed analytical method could discriminate the degradation products from the retention time of tofacitinib. The developed method was found to be selective for tofacitinib and indicated the method stability-indicating property. This can 
be applied for stability testing of tofacitinib in-process and finished products.

\section{Applicability of the developed method for skin studies of tofacitinib and dermatokinetic assessment}

The appropriateness of the method was confirmed in dermatokinetics studies on topical administration of tofacitinib. The half-life for tofacitinib was found to be $6 \mathrm{~h}$. The trapezoidal rule was employed for the calculation of AUC 0-last for tofacitinib. Considering the obtained results, the proposed and validated method could be widely used for their routine analysis and for understanding the in vivo dermatokinetics studies.

\section{Applicability in characterization of nanoformulation}

The applicability of the validated analytical method was appraised by determining the percentage entrapment efficiency, cumulative drug release of tofacitinib from the SLNs, stability, and estimation of tofacitinib from the skin tissues. Tofacitinib-loaded SLNs were analyzed in triplicate for the entrapment efficiency. The entrapment efficiency of tofacitinib was found to be $96.44 \pm 0.62 \%$. Further, the stability of the prepared nanoparticles was determined by estimating the entrapment efficiency of tofacitinib-loaded SLNs after 3 months. The entrapment efficiency after 3 months was found to be $76.37 \pm 1.31 \%$, whereas controlled samples showed $99.65 \%$ of the initial sample. The validated method was successfully determined the change in the entrapment efficiency of the formulation. Thus, this method can be used to estimate the shelf life of tofacitinib in nanoformulation. The in vitro release is acquiring considerable interest as a surrogate test for product capability. The in vitro drug release samples were collected and filtered through the $0.22-\mu$ membrane, and the concentration of tofacitinib was determined by the developed method $(n=3)$. The cumulative in vitro drug release from nanoparticles was found to be $34.45 \pm 1.65 \%$ at $6 \mathrm{~h}$. The developed method appropriately quantified the tofacitinib in the lipid nanoformulations from the first hour until the end of the release study. The total amount of drug was found to be 98.9\%, and the unreleased drug from SLNs was found to be $64.55 \pm 0.98 \%$. The developed method showed good agreement for the characterization of drug release in dissolution media. Therefore, the developed method can be used for the routine assessment of the effect of formulation factors and quality control. The skin tissue samples were analyzed in six replicates for the determination of \% recovery of tofacitinib. The \% recovery of tofacitinib from the skin tissue was found to be $95.61 \pm 0.96$, with peak purity of $99.99 \pm 0.54 \%$. A specific peak was observed at 6.1 min without any interference with the skin matrix. The obtained results confirmed that the developed method can be applied for the quantification of tofacitinib during skin permeation and retention studies, dermatokinetics studies.

\section{Discussion}

The aim of this research work is to improve and validate the RP-HPLC method for estimating tofacitinib in nanoparticles and dermatokinetics studies. The preliminary trials revealed that the peak properties were mostly influenced by the percentage of organic phase and $\mathrm{pH}$ of the aqueous phosphate buffer. Using the principle of optimization, we set out to improve the process. The application of the DoE approach allowed for a systematic and easy screening of method variables [38]. Response surface methodology-based $3^{2}$ factorial design was used to confirm the optimum organic phase composition in mobile phase, buffer $\mathrm{pH}$ for the analytical method and to ascertain a mathematical relationship between variables and responses (tailing factor and retention time) [39]. The tailing factor was decreased with an increase in methanol percentage in mobile phase composition, while the $\mathrm{pH}$ of phosphate buffer also showed a significant decrease in the tailing factor [40].

The optimized chromatogram conditions exhibited retention time (6.1 $\mathrm{min})$, which is a time-saving RP-HPLC method. The analytical methods involving expensive high-cost equipment complicated sample preparation procedures are difficult to apply in routine laboratories $[10,14,15]$. However, the previously reported spectrometric methods could not confirm the sensitivity in nanogram level, peak purity, and tofacitinib-specific chemical stability. The current validated method has additional advantages over previously reported methods such as short run time, low flow rate, and simple sample preparation procedure and validated as per $\mathrm{ICH}$ guidelines with respect to stability-indicating property, robustness, and specificity $[20,25]$. Intraday and interday precision at all QC levels of tofacitinib showed that the \% RSD of developed methods were in the range of $1.1666 \pm 0.019 \%$ to $1.7295 \pm 0.0423 \%$ and $1.2219 \pm 0.014 \%$ to $1.8017 \pm 0.032 \%$, respectively. The \% bias on intraday and interday was found in the range of $0.818 \pm 0.39 \%-$ $1.908 \pm 0.20 \%$ and $0.824 \pm 0.39-1.685 \pm 0.43 \%$, respectively. The acceptable values of \% RSD and \% bias less than $2 \%$ indicated that the validated RP-HPLC method is reliable and precise and is in excellent accordance with the regulatory guidelines [41].

The obtained LOD and LOQ values were found to be 16.5 and $49.5 \mathrm{ng} / \mathrm{mL}$ indicating that this method has a higher sensitivity than previously reported RP-HPLC methods for tofacitinib [16]. The specificity study confirmed that the developed analytical method could quantify tofacitinib in the presence of formulation excipients. 
The appropriateness of the method was also confirmed in dermatokinetics studies on topical administration of tofacitinib [42]. Finally, the validated method was applied to quantify tofacitinib during solid lipid nanoparticle characterization, skin retention studies, and dermatokinetics studies [43]. The method quantified the tofacitinib in skin layers after application of topical formulation. After topical application of (350 mg) conventional cream, the epidermal concentration and dermal concentration of tofacitinib were slowly reached to $C_{\max }$ of $9.88 \mu \mathrm{g} / \mathrm{cm}^{2}$ and $19.95 \mu \mathrm{g} / \mathrm{cm}^{2}$, respectively, at $6 \mathrm{~h}\left(t_{\max }\right)$. The AUC $0-\infty$ in epidermal and viable skin layers was found to be $224.78 \mu \mathrm{g} / \mathrm{cm}^{2}$ and $345.04 \mu \mathrm{g} / \mathrm{cm}^{2}$, respectively. Based on the findings, the proposed and validated method could be widely utilized for regular analysis and to quantify tofacitinib in in vivo dermatokinetics experiments [44].

\section{Conclusion}

A simple, sensitive, reproducible, robust, cost-effective stability-indicating analytical method has been developed effectively using the Design of experiments for quantification of tofacitinib in pharmaceutical conventional dosage forms and nanoformulations. The optimized chromatographic conditions were fully validated as per ICH Q2 (R1) guidelines and found to be economical for the routine analysis in laboratory conditions compared to the reported LC-MS and HPLC methods. The stabilityindicating studies showed distinct peaks from drug peak during the acid, base, oxidative, and thermal hydrolysis conditions. The validated analytical method evidenced its utility in the estimation of tofacitinib in the pharmaceutical nanoformulations and different skin layers (viable epidermis and dermis). Thus, the developed method can be utilized for industry and academia for tofacitinibloaded nanoformulations characterization and stability evaluation.

\begin{abstract}
Abbreviations
USFDA: United States Food and Drug Administration; TYK2: Tyrosine kinase; LC-MS/MS: Liquid chromatography with tandem mass spectrometry; RPHPLC: Reverse-phase high-performance liquid chromatography; SLNs: Solid lipid nanoparticles; DoE: Design of experiment; QC: Quality control; LLOQ: Lower limit of quantification; LOD: Limit of detection; LOQ: Limit of quantification; S/N: Signal to noise; RSD: Relative standard deviation; PRESS: Prediction error sum of squares; ANOVA: Analysis of variance.
\end{abstract}

\section{Acknowledgements \\ Ph.D. fellowship from DST-INSPIRE, Govt. of India [\#IF190259], is gratefully acknowledged.}

\section{Plant authentication}

Not applicable.

\section{Authors' contributions}

SG is the methodology, investigation, and writing —original draft; RNS is the conceptualization, resources; GS is the conceptualization, investigation, project supervision, writing-review and editing. All authors read and approved the final manuscript.

\section{Funding}

No funding was received to assist with the preparation of this manuscript.

Availability of data and materials

Data and material are available upon request.

\section{Declarations}

Ethics approval and consent to participate

The article does not contain any studies with human or animal subjects performed by any of the authors.

\section{Consent for publication}

Not applicable.

\section{Competing interests}

No competing interests.

\section{Author details}

${ }^{1}$ Industrial Research Laboratory, Department of Pharmacy, Birla Institute of Technology and Science (BITS), Pilani, Pilani Campus, Pilani, Rajasthan 333031, India. ${ }^{2}$ Birla Institute of Technology and Science (BITS), Pilani, Dubai

Campus, Dubai, UAE.

Received: 10 May 2021 Accepted: 22 August 2021

Published online: 30 August 2021

\section{References}

1. Gorantla S, Singhvi G, Rapalli VK, Waghule T, Dubey SK, Saha RN (2020) Targeted drug-delivery systems in the treatment of rheumatoid arthritis: recent advancement and clinical status. Ther Deliv 11(4):269-284. https:// doi.org/10.4155/tde-2020-0029

2. Fragoulis GE, Mcinnes IB, Siebert S (2019) JAK-inhibitors. New players in the field of immune-mediated diseases, beyond rheumatoid arthritis. Rheumatology 58:143-154

3. Li ZG, Liu Y, Xu HJ, Chen ZW, De BC, Gu JR, Zhao DB (2018) Efficacy and safety of tofacitinib in Chinese patients with rheumatoid arthritis. Chin Med J 131(22):2683

4. Xeljanz. Available from https://www.ema.europa.eu/en/documents/asses sment-report/xeljanz-epar-public-assessment-report_en.pdf

5. Hosking A-M, Juhasz M, Mesinkovska NA (2018) Topical Janus kinase inhibitors: a review of applications in dermatology. J Am Acad Dermatol 79(3):535-544

6. Singhvi G, Patil S, Girdhar V, Dubey SK (2019) Nanocarriers for topical drug delivery: approaches and advancements. Nanosci Nanotechnol Asia 9(3):329-336

7. Girdhar V, Patil S, Banerjee S, Singhvi G (2018) Nanocarriers For drug delivery: mini review. Curr Nanomed 8(2):88-99

8. Wu X, Zeng X, Wang L, Hang T, Song M (2017) Identification of related substances in tofacitinib citrate by LC-MS techniques for synthetic process optimization. J Pharm Biomed Anal 143:17-25

9. Sharma K, Giri K, Dhiman V, Dixit A, Zainuddin M, Mullangi R (2015) A validated LC-MS/MS assay for simultaneous quantification of methotrexate and tofacitinib in rat plasma: application to a pharmacokinetic study. Biomed Chromatogr 29(5):722-732

10. Kadi AA, Abdelhameed AS, Darwish HW, Attwa MW, Bakheit AH (2016) Liquid chromatographic-tandem mass spectrometric assay for simultaneous quantitation of tofacitinib, cabozantinib and afatinib in human plasma and urine. Trop J Pharm Res 15(12):2683-2692

11. Bharwad KD, Shah PA, Shrivastav PS, Singhal P (2019) Development and validation of a rapid and sensitive UPLC-MS/MS assay for the quantification of tofacitinib in human plasma. Biomed Chromatogr 33(4):e4458

12. Christmann $R$, Ho DK, Wilzopolski J, Lee S, Koch M, Loretz B et al (2020) Tofacitinib loaded squalenyl nanoparticles for targeted follicular delivery in inflammatory skin diseases. Pharmaceutics 12(12):1-17 
13. Liu P, Wu S, Dong Z (2021) Identification of the metabolites of tofacitinib in liver microsomes by liquid chromatography combined with high resolution mass spectrometry. Biomed Chromatogr 35(6):e5081

14. Dixit A, Mallurwar SR, Sulochana SP, Zainuddin M, Mullangi R (2019) Determination of tofacitinib in mice whole blood on dried blood spots using LC-ESI-MS/MS: application to pharmacokinetic study in mice. Drug Res 69(6):330-336

15. Handler AM, Pommergaard Pedersen G, Troensegaard Nielsen K, Janfelt C, Just Pedersen A, Clench MR (2021) Quantitative MALDI mass spectrometry imaging for exploring cutaneous drug delivery of tofacitinib in human skin. Eur J Pharm Biopharm 159:1-10

16. Naik P, Chandra Sekhar KB (2018) A novel stability indicating chromatographic method development and validation for the quantification of tofacitinib in pure and its dosage form. IOSR J Appl Chem 11(2):33-37

17. Siva B, Kiran S, Raja S (2018) RP-HPLC method development and validation for the quantification of tofacitinib. J Life Sci Res 4(4):646-653

18. Dowty ME, Jesson MI, Ghosh S, Lee J, Meyer DM, Krishnaswami S et al (2014) Preclinical to clinical translation of tofacitinib, a Janus kinase inhibitor. Rheumatoid Arthritis J Pharmacol Exp Ther 348(1):165-173

19. Paniagua R, Campbell A, Changelian PS, Reitz BA, Prakash C, Borie DC (2005) Quantitative analysis of the immunosuppressant CP-690,550 in whole blood by column-switching high-performance liquid chromatography and mass spectrometry detection. Ther Drug Monit 27(5):608-616

20. Kim JE, Park MY, Kim SH (2020) Simple determination and quantification of tofacitinib, a JAK inhibitor, in rat plasma, urine and tissue homogenates by HPLC and its application to a pharmacokinetic study. J Pharmaceut Investig 50(6):603-612. https://doi.org/10.1007/s40005-020-00490-

21. Sankar ASK, Datchayani B, Balakumaran N, Rilwan M, Subaranjani R (2017) Development of a validated reverse phase liquid chromatographic assaymethod for determination of tofacitinib in pure form and in physical admixtures. Res J Pharm Technol 10(1):223-226

22. Vk S, Dhiman V, Giri KK, Sharma K, Zainuddin M, Mullangi R (2015) Development and validation of a RP-HPLC method for the quantitation of tofacitinib in rat plasma and its application to a pharmacokinetic study. Biomed Chromatogr 29(9):1325-1329

23. Bashir S, Aamir M, Sarfaraz RM, Hussain Z, Sarwer MU, Mahmood A et al (2021) Fabrication, characterization and in vitro release kinetics of tofacitinib-encapsulated polymeric nanoparticles: a promising implication in the treatment of rheumatoid arthritis. Int J Polym Mater Polym Biomater 70(7):449-458. https://doi.org/10.1080/00914037.2020.1725760

24. Gorantla S, Saha RN, Singhvi G (2021) Spectrophotometric method to quantify tofacitinib in lyotropic liquid crystalline nanoparticles and skin layers: application in ex vivo dermal distribution studies. Spectrochim Acta Part A Mol Biomol Spectrosc 255:119719

25. Sankar ASK, Shanmugasundaram P, Datchayani B, Balakumaran N, Rilwan M, Subaranjani R et al (2017) Stress degradation studies and development of validated spectrometric-assay-method for determination of tofacitinib in pure and physical admixtures. Res J Pharm Technol 10(1):117-120

26. Kushner J, Lamba M, Stock T, Wang R, Nemeth MA, Alvey C et al (2020) Development and validation of a Level $A$ in vitro in vivo correlation for tofacitinib modified-release tablets using extrudable core system osmotic delivery technology. Eur J Pharm Sci 147:105200. https://doi.org/ 10.1016/j.ejps.2019.105200

27. Patel PN, Karakam VS, Samanthula G, Ragampeta S (2015) Quality-bydesign-based ultra high performance liquid chromatography related substances method development by establishing the proficient design space for sumatriptan and naproxen combination. J Sep Sci 38(19):33543362. https://doi.org/10.1002/jssc.201500343

28. Shukla SK, Kadry H, Bhatt JA, Elbatanony R, Ahsan F, Gupta V (2020) Statistical optimization and validation of a novel ultra-performance liquid chromatography method for estimation of nintedanib in rat and human plasma. Bioanalysis 12(3):159-174. https://doi.org/10.4155/bio-2019-0284
29. Krishna KV, Saha RN, Puri A, Viard M, Shapiro BA, Dubey SK (2019) Pre-clinical compartmental pharmacokinetic modeling of 2-[1-hexyloxyethyl]2-devinyl pyropheophorbide-a (HPPH) as a photosensitizer in rat plasma by validated HPLC method. Photochem Photobiol Sci 18(5):1056-1063

30. Singhvi G, Gampa G, Saha NR (2013) Development and validation of a stability indicating liquid chromatographic method for the determination of milnacipran in bulk and its formulations. Curr Pharm Anal 9(2):191-198

31. Rajadhyaksha NS, Jain SP, Amin PD (2007) Analytical letters carbamazepine: stability indicating HPLC assay method carbamazepine-stability indicating HPLC assay method. Anal Lett 40(13):2506-2514

32. Rapalli VK, Singhvi G, Gorantla S, Waghule T, Dubey SK, Saha RN et al (2019) Stability indicating liquid chromatographic method for simultaneous quantification of betamethasone valerate and tazarotene in in vitro and ex vivo studies of complex nanoformulation. J Sep Sci 42(22):3413-3420

33. Blessy M, Patel RD, Prajapati PN, Agrawal YK (2014) Development of forced degradation and stability indicating studies of drugs-a review. J Pharm Anal 4(3):159-165

34. Rapalli VK, Kaul V, Gorantla S, Waghule T, Dubey SK, Pandey MM et al (2020) UV spectrophotometric method for characterization of curcumin loaded nanostructured lipid nanocarriers in simulated conditions: method development, in vitro and ex vivo applications in topical delivery. Spectrochim Acta Part A Mol Biomol Spectrosc 224:117392

35. Jain S, Krishna Cherukupalli S, Mahmood A, Gorantla S, Krishna Rapalli V, Kumar Dubey S et al (2019) Emerging nanoparticulate systems: preparation techniques and stimuli responsive release characteristics. J Appl Pharm Sci 9(08):130-143

36. Jain S, Mistry MA, Swarnakar NK (2011) Enhanced dermal delivery of acyclovir using solid lipid nanoparticles. Drug Deliv 1(5):395

37. Younis US, Vallorz E, Addison KJ, Ledford JG, Myrdal PB (2019) Preformulation and evaluation of tofacitinib as a therapeutic treatment for asthma. AAPS PharmSciTech 20(5):1-11

38. Ganorkar SB, Shirkhedkar AA (2017) Design of experiments in liquid chromatography (HPLC) analysis of pharmaceuticals: analytics, applications, implications and future prospects. Rev Anal Chem 36(3):20160025. https://doi.org/10.1515/revac-2016-0025/html

39. Bhargav E, Chaithanya Barghav G, Padmanabha Reddy Y, Pavan Kumar C, Ramalingam P, Haranath C (2020) A Design of Experiment (DoE) based approach for development and optimization of nanosuspensions of telmisartan, a BCS class II antihypertensive drug. Future J Pharm Sci 6(1):1-13. https://doi.org/10.1186/s43094-020-00032-2

40. Tome T (2019) Development and optimization of liquid chromatography analytical methods by using AQbD principles: overview and recent advances. Org Process Res Dev 23(9):1784-1802

41. Center for Drug Evaluation and Research (CDER) Reviewer Guidance' Validation of Chromatographic Methods (1994)

42. Rapalli VK, Sharma S, Roy A, Alexander A, Singhvi G (2021) Solid lipid nanocarriers embedded hydrogel for topical delivery of apremilast: in vitro, ex vivo, dermatopharmacokinetic and anti-psoriatic evaluation. J Drug Deliv Sci Technol 63:102442. https://doi.org/10.1016/J.JDDST.2021. 102442.

43. Thotakura N, Kumar P, Wadhwa S, Raza K, Katare P (2017) Dermatokinetics as an important tool to assess the bioavailability of drugs by topical nanocarriers. Curr Drug Metab 18(5):404-411

44. Rapalli VK, Singhvi G (2020) Dermato-pharmacokinetic: assessment tools for topically applied dosage forms. Expert Opin Drug Deliv 18:423-426. https://doi.org/10.1080/17425247.2021.1856071

\section{Publisher's Note}

Springer Nature remains neutral with regard to jurisdictional claims in published maps and institutional affiliations. 